\title{
BMJ Open Can exercise suppress tumour growth in advanced prostate cancer patients with sclerotic bone metastases? A randomised, controlled study protocol examining feasibility, safety and efficacy
}

\author{
Nicolas H Hart, ${ }^{1}$ Robert U Newton, ${ }^{1}$ Nigel A Spry, ${ }^{1,2,3}$ Dennis R Taaffe, ${ }^{1}$ \\ Suzanne K Chambers, ${ }^{1,4}$ Kynan T Feeney, ${ }^{1,5,6}$ David J Joseph, ${ }^{1,2,3}$ \\ Andrew D Redfern, ${ }^{7,8}$ Tom Ferguson, ${ }^{7}$ Daniel A Galvão ${ }^{1}$
}

To cite: Hart NH, Newton RU, Spry NA, et al. Can exercise suppress tumour growth in advanced prostate cancer patients with sclerotic bone metastases? A randomised, controlled study protocol examining feasibility, safety and efficacy. BMJ Open 2017;7:e014458. doi:10.1136/ bmjopen-2016-014458

- Prepublication history and additional material are available. To view these files please visit the journal online (http://dx.doi. org/10.1136/bmjopen-2016014458).

Received 26 September 2016 Revised 10 February 2017 Accepted 20 March 2017

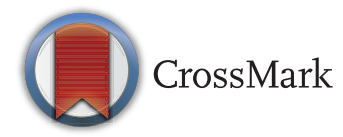

For numbered affiliations see end of article.

Correspondence to

Dr Nicolas H Hart;

n.hart@ecu.edu.au

\section{ABSTRACT}

Introduction Exercise may positively alter tumour biology through numerous modulatory and regulatory mechanisms in response to a variety of modes and dosages, evidenced in preclinical models to date. Specifically, localised and systemic biochemical alterations produced during and following exercise may suppress tumour formation, growth and distribution by virtue of altered epigenetics and endocrine-paracrine activity. Given the impressive ability of targeted mechanical loading to interfere with metastasis-driven tumour formation in human osteolytic tumour cells, it is of equal interest to determine whether a similar effect is observed in sclerotic tumour cells. The study aims to (1) establish the feasibility and safety of a combined modular multimodal exercise programme with spinal isometric training in advanced prostate cancer patients with sclerotic bone metastases and (2) examine whether targeted and supervised exercise can suppress sclerotic tumour growth and activity in spinal metastases in humans.

Methods and analysis A single-blinded, two-armed, randomised, controlled and explorative phase I clinical trial combining spinal isometric training with a modular multimodal exercise programme in 40 men with advanced prostate cancer and stable sclerotic spinal metastases. Participants will be randomly assigned to (1) the exercise intervention or (2) usual medical care. The intervention arm will receive a 3-month, supervised and individually tailored modular multimodal exercise programme with spinal isometric training. Primary endpoints (feasibility and safety) and secondary endpoints (tumour morphology; biomarker activity; anthropometry; musculoskeletal health; adiposity; physical function; quality of life; anxiety; distress; fatigue; insomnia; physical activity levels) will be measured at baseline and following the intervention. Statistical analyses will include descriptive characteristics, $\mathrm{t}$-tests, effect sizes and two-way (group $\times$ time) repeatedmeasures analysis of variance (or analysis of covariance) to examine differences between groups over time. The data-set will be primarily examined using an intention-to-
Strengths and limitations of this study

- This is a novel, phase I randomised controlled trial in humans exploring the preliminary effects of targeted exercise on tumour morphology and circulating metastatic tumour biomarkers using a sclerotic skeletal metastases model in patients with prostate cancer.

- The study is principally aimed at establishing feasibility and safety, and may lack statistical power or a suitable length of intervention to determine the efficacy of exercise on tumour biology and metastatic tumour biomarkers.

treat approach with multiple imputations, followed by a secondary sensitivity analysis to ensure data robustness using a complete cases approach.

Ethics and dissemination Ethics approval was obtained from the Human Research Ethics Committee (HREC) of Edith Cowan University and the Sir Charles Gairdner and Osborne Park Health Care Group. If proven to be feasible and safe, this study will form the basis of future phase II and III trials in human patients with advanced cancer. To reach a maximum number of clinicians, practitioners, patients and scientists, outcomes will be disseminated through national and international clinical, conference and patient presentations, as well as publication in highimpact, peer-reviewed academic journals.

Trial registration number ACTRN 12616000179437.

\section{INTRODUCTION}

Bone is the most common location for metastatic prostate carcinoma, with skeletal lesions identified in $>80 \%$ of patients with advanced prostate $_{\text {cancer. }}{ }^{1-5}$ These lesions predominantly present as sclerotic (osteoblastic) and primarily reside in the axial skeleton (spine, 
pelvis or ribs), ${ }^{6-8}$ presenting a considerable challenge for practitioners to deliver exercise interventions to this population. ${ }^{910}$ In particular, advanced cancer patients have historically been excluded from exercise intervention studies and community-based supervised exercise programmes due to potential adverse skeletal events. Consequently, patients with advanced cancer often fail to receive the crucial benefits of exercise in the management of their disease; that is, to reduce treatment toxicities, delay disease progression and increase survival through neoadjuvant, adjuvant, synergistic and targeted applications. Clinically, bone metastases present as a major concern with patients experiencing severe bone pain, increased risk of skeletal complications, spinal compression, hypercalcaemia and decreased physical function and quality of life. ${ }^{9-13}$ Subsequently, patients with metastatic bone disease experience significant morbidity with many barriers to exercise participation, thus strategies to safely and effectively reduce the burden of bone metastatic disease have a high degree of clinical importance.

Despite the unequivocal benefits of exercise for patients with cancer, ${ }^{14-25}$ fear of adverse skeletal events has led to reduced uptake by patients, and reduced referrals to exercise programmes by clinicians ${ }^{9}{ }^{10}{ }^{18}$; a cycle which exacerbates musculoskeletal fragility and tumour progression. In recent times, Galvão and colleagues ${ }^{9} 1021$ designed a modular, multimodal exercise programme (M3EP) to provide this population with safe and effective exercise modified to avoid direct loading of lesion sites; the first of its kind in exercise oncology. Preliminary findings have been promising, with exercise shown to be well tolerated (safe and efficacious), delivering clear improvements in physical function, physical activity levels and lean mass in these patients. ${ }^{10}{ }^{21}$ However, it is not yet known whether direct loading of lesion sites in bone metastases is safe to deliver, or therapeutically advantageous in providing adjuvant, synergistic or independent health benefits or tumorous modulations in patients with advanced prostate cancer.

Exercise positively alters tumour biology via numerous mechanisms in response to a variety of modes and dosages. ${ }^{26-33}$ Specifically, exercise regulates endocrine-paracrine activity, immune system function, blood glucose levels, blood cholesterol levels, insulin response and body composition ${ }^{34-41}$, while epigenetically modulating tumour cell proliferation, telomere length, telomere enzyme activity, tumour vascularity, oxidative stress capacity, platelet cloaking and platelet adhesion. ${ }^{26-3342-47}$ Although these regulatory and modulatory outcomes interact, the ability to suppress tumour formation, growth and spread by virtue of altered epigenetics and endocrine-paracrine activity through exercise is of particular interest. This emerging field of mechanomics in exercise oncology (biological alterations driven from biomechanical stimuli) ${ }^{48-51}$ presents practitioners with a unique opportunity to potentially suppress metastatic prostate carcinoma in bone through targeted exercise interventions.
Direct loading of animal metastatic bones containing human breast cancer tumour cells supports this new direction, successfully interfering with metastasis-driven tumour formation ${ }^{50}$ by delivering suppressive localised modulatory changes to the tumour microenvironment, while preserving bone material, structure and strength. ${ }^{49}$ Given the impressive ability of mechanical loading to epigenetically interfere with metastasis-driven tumour formation in osteolytic tumour cells, it is of equal interest to determine whether mechanical loading is also able to alter metastasis-driven tumour formation in sclerotic tumour cells. This may also prove to be an effective adjuvant intervention to alleviate bone pain, preserve musculoskeletal strength and suppress sclerotic tumour expansion. To our knowledge, no studies have directly targeted skeletal metastases in advanced cancer patients through controlled, localised exercise programmes in order to plausibly modulate tumour biology and suppress tumour growth in humans; nor are we aware of any studies that have specifically established the feasibility or safety of loading skeletal sites with sclerotic bone metastases in patients with advanced prostate cancer.

The aim of this study is to (1) examine whether a modular multimodal exercise programme with spinal isometric training is feasible and safe in patients with advanced prostate cancer and sclerotic bone metastases in order to produce targeted and localised adaptations surrounding spinal lesions and (2) examine the preliminary efficacy of targeted and supervised exercise to suppress sclerotic tumour growth and activity in spinal metastases in humans. We hypothesise that the exercise programme will be feasible and safe to deliver to patients with advanced prostate cancer; and that the exercise programme will help reduce the rate of sclerotic tumour progression. In addition, we also hypothesise the exercise programme will alleviate bone pain; not increase the incidence of skeletal fractures; act to preserve muscle and bone; improve physical fitness and functional ability; reduce cancer-related fatigue and increase quality of life and psychosocial well-being. The outcomes of this trial will be used to improve clinical knowledge pertaining to exercise prescription for advanced cancer patients with metastatic prostate carcinoma, specifically promoting isometric loading of bones with sclerotic lesions. The outcomes of this trial will also be used to establish preliminary efficacy of tumour suppression through exercise, thereby informing phase II and III clinical trials to help establish new clinical exercise guidelines in this high-risk and unique population.

\section{METHODS AND ANALYSIS}

\section{Study design}

This is a single-blinded (investigators blinded to group allocation), two-armed, randomised and controlled (exercise vs usual care) explorative phase I clinical trial which will examine the feasibility, safety and preliminary efficacy of combining spinal isometric training with a modulatory, 


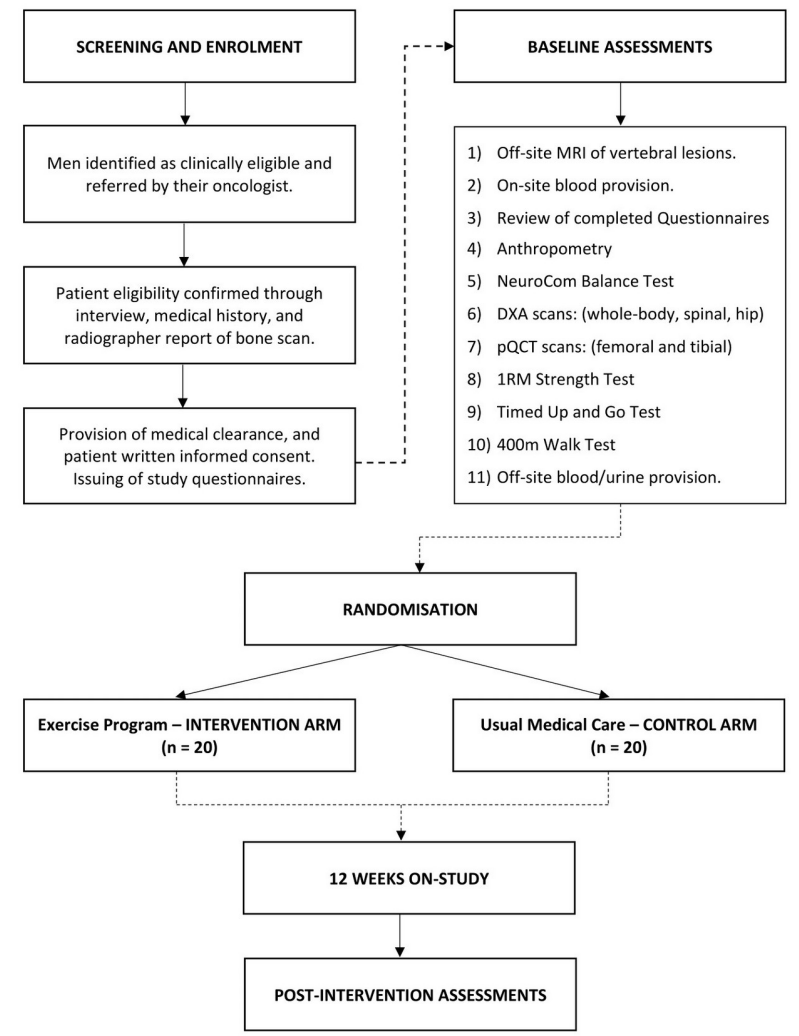

Figure 1 Schematic overview of the study protocol. DXA, dual-energy X-ray absorptiometry; PQCT, peripheral quantitative $\mathrm{CT}$; $1 \mathrm{RM}$, one repetition maximum.

multimodal exercise programme (M3EP-SIT) in men with advanced prostate cancer and sclerotic spinal bone metastases. The exercise group (intervention arm) will receive an individually tailored and supervised 3-month (12 weeks) exercise programme involving resistance, aerobic, flexibility and isometric exercises in addition to usual medical care. The control group will receive usual medical care during this time and will be asked not to change their baseline levels of physical activity. Following the trial, the control group will be offered the same exercise programme if the intervention is deemed to be safe and feasible. This procedure has been shown to be an effective strategy to minimise study contamination, patient withdrawals or loss of patients to follow-up in prior exercise oncology trials. ${ }^{9439}$

\section{Recruitment}

Patients will be recruited by invitation of their cancer specialist (urologist, radiation oncologist or medical oncologist) who will provide clinically eligible patients with a study information sheet and refer these patients to a study coordinator. If patients are interested in participation, and their eligibility is confirmed, they will receive an informed consent document to read and sign in the presence of a study investigator and clinical research coordinator before undertaking baseline measurements prior to randomisation (figure 1).

\section{Randomisation}

Patients will be randomly allocated in a ratio of $1: 1$ to the two study arms: exercise or usual care, stratified by age ( $\leq 70$ years, $>70$ years) and time since completion of chemotherapy, radiotherapy to spinal metastases, or change in hormone therapy ( $<3$ months, $\geq 3$ months) for approximate balance between groups in order to mitigate confounding factors pertaining to variations due to ageing (ie, differences in physical function, sarcopenia, osteopenia and osteoporosis) and to account for variations in the treatment and washout effect of any recent completions of chemotherapy and radiotherapy, or changes in hormone therapy (ie, if progressed to secondline antiandrogen agents). All patients will be required to be on androgen deprivation therapy (ADT) prior to, and during this study, as per standard of care for this patient population. A research officer with no patient contact will be responsible for randomisation of patients into either group by using a computer random assignment programme. Study investigators and exercise physiologists conducting testing procedures will be blinded to group allocation. Only exercise physiologists not in the research team will be permitted to deliver the exercise intervention to participants in order to maintain integrity of the blinding process.

\section{Participants}

Forty men (20 subjects per arm) with prostate cancer and stable bone metastases in cervical, thoracic and/or lumbar vertebrae, who have not engaged in regular exercise (ie, undertaking structured aerobic or resistance training two or more times per week) within the past three months will serve as participants. Due to the novelty of this explorative phase I clinical trial with a primary endpoint to establish feasibility and safety, our sample size was chosen based on prior preclinical animal studies, ${ }^{26} 303250$ human pilot studies $^{31}{ }^{33}$ and consideration of recruitment ability for advanced prostate cancer patients with bone metastases. In addition, to demonstrate a meaningful difference (effect size $\geq 1.0 ; 80 \%$ power; alpha level of 0.05 (two-tailed)) for most secondary endpoints, 16 subjects per group would be required. To account for up to a $25 \%$ attrition rate, 40 subjects will be randomised evenly to each study arm (exercise: $n=20$; control: $n=20$ ) to assist with establishing preliminary efficacy of secondary endpoints and to form the basis of sophisticated power calculations for future phase II and III randomised controlled trials.

Patients will be excluded from this trial if they have commenced chemotherapy, if they are receiving radiotherapy for any spinal bone metastases, if they have commenced or changed hormone therapy within 3 months of enrolment or are currently receiving any other experimental treatments or non-approved therapies. Patients are permitted to receive radiotherapy for non-spinal bone metastases while enrolled in this trial. Patients require medical clearance prior to enrolment, therefore they must achieve an Eastern Cooperative Oncology Group performance status of $\leq 1$; and must not 
have any acute illness, significant bone pain or cardiovascular or neurological disorders that could inhibit exercise participation. All participants must provide written informed consent prior to participation. The protocol has been approved by the Human Research Ethics Committee of Edith Cowan University (ID: 13399 HART and 14146 HART); and Sir Charles Gairdner and Osborne Park Health Care Group (ID: 2016-118). This trial is also registered with the Australia and New Zealand Clinical Trails Register (ANZCTR), trial ID: ACTRN-12616000179437. All data relevant to the study will be kept on password-encrypted computers accessible by study investigators situated at the Exercise Medicine Research Institute (Perth, WA, Australia).

\section{Measurements}

Primary and secondary endpoints will be assessed at baseline (week 0 ), postintervention (week 13) and throughout the 12-week on-trial period (table 1).

\section{Primary endpoint}

\section{Feasibility}

Feasibility will be quantified through a series of multiitem categories including patient recruitment and trial completion; patient safety; programme tolerance; programme adherence and programme compliance (table 2). Programme safety will be assessed by recording the incidence and severity of adverse events and/or skeletal complications throughout the on-trial period for intervention and control arms. Skeletal complications include heightened pain at sites of bone metastases and/ or pathological skeletal fractures. The nature, severity and impact of bone pain will be examined using the FACIT Bone Pain questionnaire at baseline and post intervention.

Programme tolerance, adherence and compliance will be assessed for the intervention arm only. Specifically, programme tolerance will be quantified by measuring presessional bone pain and fatigue at each exercise session through visual analogue scales (VAS, 0-10) and by recording sessional rating of perceived exertion (Borg Scale, 0-10) and sessional tolerance (VAS, 0-7) after each exercise session.

Programme adherence and compliance will be assessed using an exercise diary completed by the patient at all clinic-based and home-based exercise sessions to record the volume of resistance training (weight lifted $(\mathrm{kg})$, sets and repetitions), aerobic training (intensity (level), duration (minutes), speed (revolutions-per-minute), heart rate (maximum and average) and rating of perceived exertion) flexibility training (repetitions) and isometric training (repetitions and hold duration) completed. These data will be compared with the prescribed and individualised exercise programme provided to each patient in order to establish programme adherence (completed vs missed sessions) and compliance (prescribed vs actual exercise completed for each training modality (resistance, aerobic, flexibility and isometric)).

\section{Secondary endpoints}

\section{Tumour morphology}

Tumour morphology will be measured using axial T1-weighted MRI scans (1.5T, Magnetom Essenza, Siemens, Victoria, Australia) in locations where sclerotic lesions have been identified in patients with bone metastases at either thoracic or lumbar spinal regions. ${ }^{52-54}$ Location of metastatic lesions will be previously recorded through bone scans provided by the patient's primary oncologist prior to referral to this study. All scans will be performed on the same MRI machine by the same radiologist using a standardised sequence and routine for scout and primary acquisitions. Specifically, spinal bone metastases will be identified and confirmed using three preliminary axial scout scans in the sagittal plane to view cervical, thoracic and lumbar regions, respectively (T2-weighted; imaging frequency $=63.66 \mathrm{~Hz}$; slice thickness $=3.0-4.0 \mathrm{~mm}$; spacing between slices $=3.6-6.0 \mathrm{~mm}$; echo train length $=16-21$; flip angle $=140-150^{\circ}$; acquisition

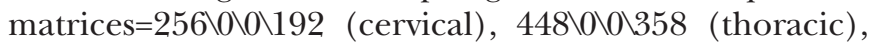
$320 \backslash 0 \backslash 0 \backslash 320$ (lumbar)).

Primary acquisition for each affected vertebrae will be performed in the transverse plane, capturing the vertebrae above and below to produce an image with three vertebrae in total (T1-weighted; imaging frequency $=63.66 \mathrm{~Hz}$; slice thickness $=4.0 \mathrm{~mm}$; space between slices $=4.2 \mathrm{~mm}$; echo train length $=3$; flip angle $=150^{\circ}$; acquisition matrix $=384 \backslash 0 \backslash 0 \backslash 307)$. Following acquisition, tumour morphology (volume $\left(\mathrm{mm}^{3}\right)$, intensity $(\mathrm{W} / \mathrm{sr}$ ) will be examined for each slice using ITK-Snap (V.3.6.0) image analysis software $^{55}$ (figure 2). All images will be examined at the conclusion of the study by two independent researchers for consistency in analysis and to establish intra-rater and inter-rater reliability coefficients.

\section{Biomarkers}

Metastatic tumour biomarkers, hypoxia-inducible factor 1-alpha (HIF-1 $\alpha$ ) and transformation growth-like factor beta (TGF- $\beta$ ) will be examined through unfasted serological samples acquired at baseline and postintervention testing sessions to measure hypoxic activity and transformation-growth activity, respectively; identified as key synergistic drivers of metastatic tumour progression. ${ }^{56}$ Fasted serological and first-void urianalytical samples will be collected within 48 hours of baseline and postintervention testing sessions to measure bone metabolic activity and systemic inflammation. Specifically, bone formation marker, amino-terminal propeptide of type 1 procollagen $(\mathrm{P} 1 \mathrm{NP})$; bone resorption marker, aminoterminal collagen type I telopeptide (NTx); bone disorder marker, alkaline phosphate (ALP); inflammation marker, $\mathrm{C}$ reactive protein (CRP) and fasting glucose and lipid profiles will be examined. Prostate-specific antigen (PSA) will also be assessed. All fasted serological and first-void urianalytical biomarkers will be collected in the morning, and assessed by the same accredited laboratory (St John of God Hospital, Pathology Laboratory, Perth, Western Australia). 
Table 1 Schedule of assessments at baseline and post intervention

\begin{tabular}{lll}
\hline Measures & Baseline & Post intervention \\
\hline $\begin{array}{l}\text { Tumour morphology (off-site) } \\
\text { MRI scan: T1-axial }\end{array}$ & $\mathrm{X}$ & $\mathrm{X}$ \\
$\begin{array}{l}\text { Tumour biomarkers } \\
\text { Blood: HIF-1 } \alpha \text {, TGF- } \beta\end{array}$ & $\mathrm{X}$ & $\mathrm{X}$ \\
Anthropometry & & \\
Height $(\mathrm{cm})$ & $\mathrm{X}$ & $\mathrm{X}$ \\
Weight $(\mathrm{kg})$ & $\mathrm{X}$ & $\mathrm{X}$ \\
Waist circumference $(\mathrm{cm})$ & $\mathrm{X}$ & $\mathrm{X}$ \\
Hip circumference $(\mathrm{cm})$ & $\mathrm{X}$ & \\
Femoral length $(\mathrm{mm})$ & $\mathrm{X}$ & $\mathrm{X}$ \\
Tibial length $(\mathrm{mm})$ & $\mathrm{X}$ & $\mathrm{X}$ \\
Body mass index $\left(\mathrm{kg} / \mathrm{m}^{2}\right)$ & $\mathrm{X}$ & $\mathrm{X}$ \\
Waist-to-hip ratio & &
\end{tabular}

Body composition

DXA scans: whole-body, spinal, hip $\quad x \quad x$

pQCT scans: femoral, tibial

$\mathrm{x}-\mathrm{x}$

Physical assessments

NeuroCom Balance test

1RM Strength test (leg extension)

$400 \mathrm{~m}$ walk test

Timed Up and Go test

$\mathrm{x}$

Other biomarkers (off-site)

Blood: P1NP, ALP, CRP, fasting glucose and lipids, PSA

Urine: NTx

$\begin{array}{ll}x & x \\ x & x \\ x & x \\ & x\end{array}$

Questionnaires

Demographic and health history $\quad \mathrm{X}$

Concomitant medications $\quad X$

Health-related Quality of Life (SF-36)

Cancer-specific Quality of Life (EORTC: QLQ30, PR25)

Brief Symptom Index (BSI-18)

Insomnia Severity Index

Godin Leisure-Time Exercise

$\begin{array}{ll}X & x \\ X & X\end{array}$

$\mathrm{X}$
$\mathrm{X}$

$x$
$x$
$x$

$\begin{array}{ll}x & x \\ x & x\end{array}$

$x-x$

$x-x$

$x \quad x$

$\mathrm{x}$

$x$

\section{Exercise programme}

Clinic exercise record sheet (prescribed vs actual)

Home exercise record sheet (prescribed vs actual)

At each exercise session

At each exercise session

ALP, alkaline phosphate; CRP, C reactive protein; DXA, dual-energy X-ray absorptiometry; EORTC, European Organisation for Research and Treatment of Cancer; HIF-1 $\alpha$, hypoxia-inducible factor 1-alpha; NTx, amino-terminal collagen type 1 telopeptide; P1NP, aminoterminal propeptide of type 1 procollagen; pQCT, peripheral quantitative CT; PSA, prostate-specific antigen; SF-36, short form-36; TGF- $\beta$, transformation growth-like factor beta.

\section{Anthropometry}

Stature will be recorded to the nearest $0.1 \mathrm{~cm}$ using a wall-mounted stadiometer (Model 222, Seca, Hamburg, $\mathrm{DE})$, with body mass recorded to the nearest $0.1 \mathrm{~kg}$ using an electronic scale (AE Adams CPW Plus-200, Adam Equipment, CT, USA). Waist and hip circumferences are defined as the midpoint between the tenth rib and iliac crest; and the level of the greater trochanter, respectively, with the waist-to-hip ratio calculated. Waist circumference and hip circumference will be measured to the nearest $0.1 \mathrm{~cm}$ using a constant-tension, retractable measuring tape (Model 4414, Tech-Med Services, NY, USA). Stature, waist circumference and hip circumference will be performed in triplicate for each participant, with the average of each variable retained for analysis. 
Musculoskeletal health

Whole-body, segmental (axial, appendicular) and regional (spinal, hip, proximal femur) scans will be performed to examine bone area, areal bone mineral content, areal bone mineral density (aBMD) and lean mass using dual-energy X-ray absorptiometry (DXA; Hologic Discovery A, Waltham, MA). Whole-body and appendicular segmentations will be analysed in accordance with Hart and colleagues. ${ }^{57}$ Regional analyses (lumbar spine, total hip, femoral neck, trochanter, Wards triangle) will be performed in accordance with Hologic's manufacturer specifications. ${ }^{58}$

Appendicular, non-lesion control sites will be scanned to quantify bone material, structure and strength using peripheral Quantitative Computed Tomography (pQCT; XCT-3000, Stratec, Pzochienheim, Germany). Specifically trabecular, cortical, marrow and total volumetric density (Tb.vBMD, Ct.vBMD, Ma.vBMD, Tt.vBMD); trabecular, cortical, marrow and total cross-sectional area (Tb.Ar, Ct.Ar, Ma.Ar, Tt.Ar); cortical thickness (Ct.Th); stress-strain index (SSIPOL); absolute fracture load (FL. $\mathrm{Ab})$ and relative fracture load (FL.Rel) of the left femur (4\% and $33 \%$ slices) and left tibia $(4 \%, 14 \%, 38 \%$ and $66 \%$ slices) will be measured and analysed in accordance with Hart and colleagues. ${ }^{59}$ Muscle cross-sectional area (Mu.Ar) will also be quantified.

\section{Adiposity}

Whole-body, segmental and central subcutaneous adipose tissue (fat mass), central visceral adipose tissue (VAT; area, mass and volume) and android to gynoid ratio will be measured using DXA. Whole-body and appendicular segmentations will be generated in accordance with Hart and colleagues. ${ }^{57}$ Fat area (Fa.Ar) and muscle density (Mu.Den) of the thigh and shank segments will be measured using pQCT, ${ }^{59}$ as an indication of subcutaneous and intramuscular fat infiltration, respectively.

\section{Objective measures of physical function}

Muscle strength, aerobic capacity and physical function will be measured through a series of assessments. Muscle strength will be measured using the one repetition maximum (1RM) test for the leg extension exercise. This exercise was chosen as it can be safely performed by all patients included in this study. The $400 \mathrm{~m}$ walk test and Timed Up and Go test will be used as measures for aerobic capacity and physical function respectively. In addition, patients will also undergo a comprehensive balance test (NeuroCom Smart Balance Master, Natus Medical, USA).

\section{Quality of life, anxiety, distress, fatigue, insomnia and physical} activity

Health-related quality-of-life outcomes for general health, pain, vitality, social functioning, emotional role and mental health will be measured by the Short Form 36 (SF-36, IQOLA) survey. In addition, the EORTC QLQ-C30 (cancer) and EORTC PR-25 (prostate cancer) surveys will also be provided to measure cancer-specific indices of
Table 2 Assessments of study feasibility

\begin{tabular}{|c|c|}
\hline Measures & Time of collection \\
\hline \multicolumn{2}{|l|}{ Recruitment and completion } \\
\hline Referred patients & Trial completion \\
\hline Eligible patients & Trial completion \\
\hline Enrolled patients & Trial completion \\
\hline Eligibility rate & Trial completion \\
\hline Recruitment rate & Trial completion \\
\hline Trial completions & Trial completion \\
\hline Patient withdrawals & Trial completion \\
\hline Patient dropouts & Trial completion \\
\hline Trial contamination & Trial completion \\
\hline \multicolumn{2}{|l|}{ Patient safety (control arm) } \\
\hline Number of adverse events & Tri-weekly record \\
\hline Severity of adverse events & Tri-weekly record \\
\hline Number of skeletal complications & Tri-weekly record \\
\hline \multicolumn{2}{|l|}{ Patient safety (intervention arm) } \\
\hline Number of adverse events & $\begin{array}{l}\text { At each exercise } \\
\text { session }\end{array}$ \\
\hline Severity of adverse events & $\begin{array}{l}\text { At each exercise } \\
\text { session }\end{array}$ \\
\hline Number of skeletal complications & $\begin{array}{l}\text { At each exercise } \\
\text { session }\end{array}$ \\
\hline \multicolumn{2}{|c|}{ Programme tolerance (intervention arm) } \\
\hline Presessional bone pain & $\begin{array}{l}\text { At each exercise } \\
\text { session }\end{array}$ \\
\hline Presessional fatigue & $\begin{array}{l}\text { At each exercise } \\
\text { session }\end{array}$ \\
\hline $\begin{array}{l}\text { Sessional rating of perceived } \\
\text { exertion }\end{array}$ & $\begin{array}{l}\text { At each exercise } \\
\text { session }\end{array}$ \\
\hline Sessional tolerance & $\begin{array}{l}\text { At each exercise } \\
\text { session }\end{array}$ \\
\hline \multicolumn{2}{|c|}{ Programme adherence (intervention arm) } \\
\hline Number of completed sessions & Post intervention \\
\hline Number of missed sessions & Post intervention \\
\hline \multicolumn{2}{|l|}{ Programme compliance (intervention arm) } \\
\hline $\begin{array}{l}\text { Prescribed versus actual exercise } \\
\text { completed (for each exercise mode) }\end{array}$ & Post intervention \\
\hline $\begin{array}{l}\text { Percent of total volume completed } \\
\text { (for each exercise mode) }\end{array}$ & Post intervention \\
\hline
\end{tabular}

quality of life. Specifically, the EORTC QLQ-C30 includes five functional scales (physical, role, emotional, cognitive and social functioning), three symptom scales (fatigue, nausea and vomiting, and pain), a global health/quality-of-life scale and six single items (dyspnoea, insomnia, appetite loss, constipation, diarrhoea and financial difficulty); whereas the EORTC PR-25 also assesses urinary symptoms, bowel symptoms, treatment-related symptoms and sexual functioning. The FACIT fatigue questionnaire will be administered to directly assess cancer-related 

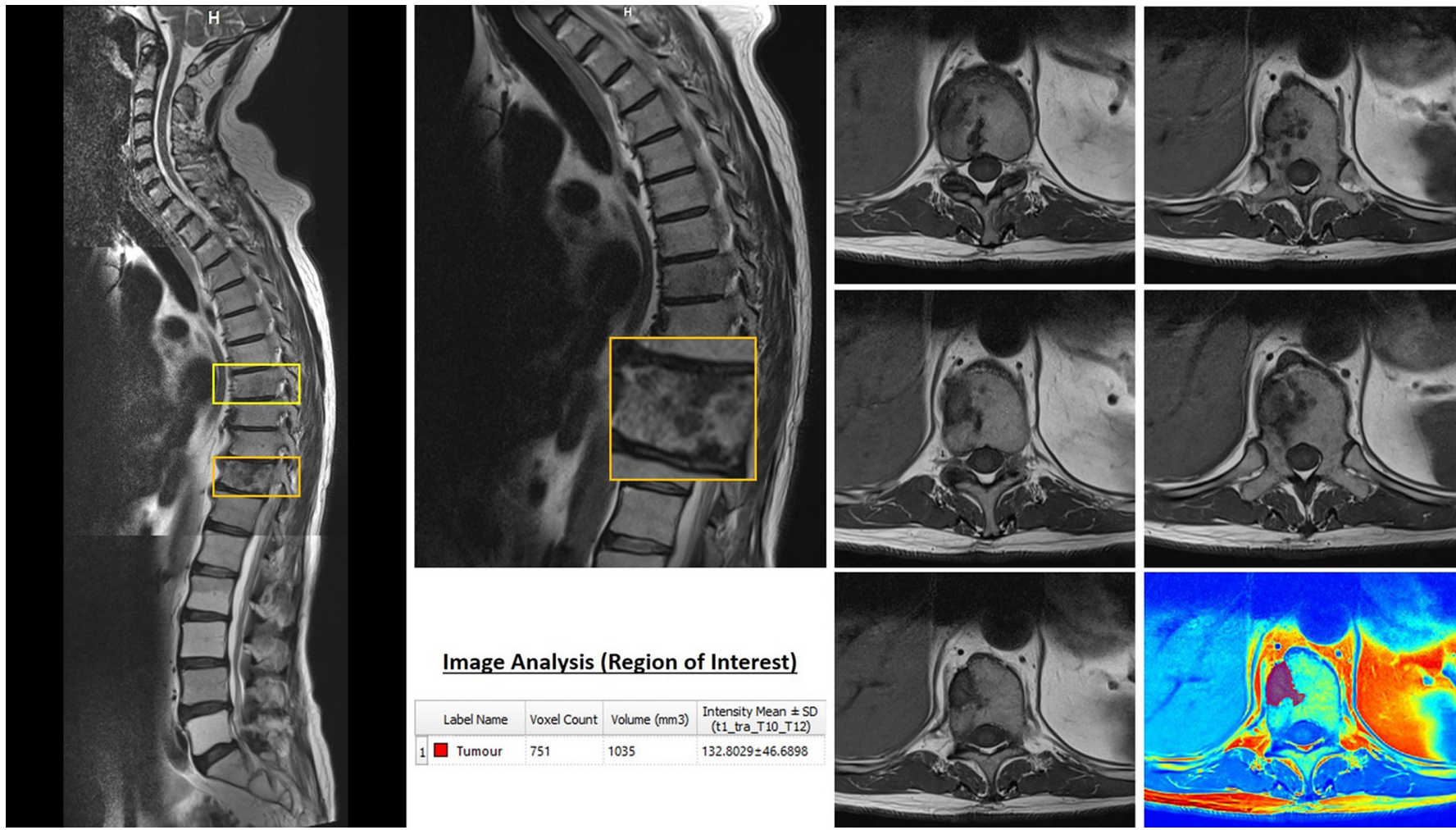

Image Analysis (Region of Interest)

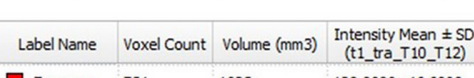
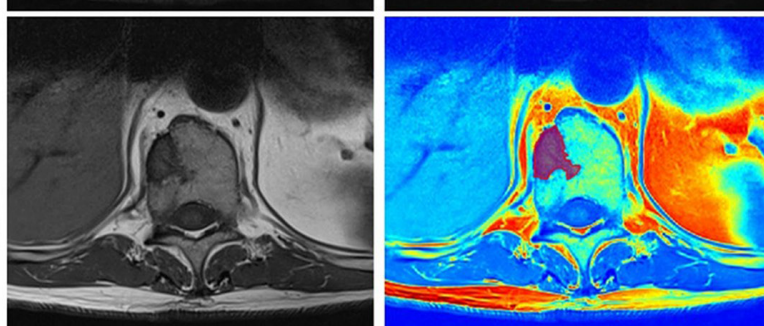

Figure 2 Example MRI image acquisition. (Left) Cervical, thoracic and lumbar scout views stitched together with two sclerotic lesions identified at T8 and T11. (Top, middle) Thoracic scout view at higher resolution with the T11 vertebrae magnified. (Bottom, middle) Example data outputs provided by regional analysis. (Right) Slice by slice, cephalad to caudal, transverse view at each level of the T11 lesion, with an example colour map and tumoural analysis of one slice in isolation.

fatigue. The Brief Symptom Inventory (BSI-18) will be used to assess psychological distress for anxiety, depression, somatisation and global distress severity domains. The Insomnia Severity Index (ISI) will be used to measure sleep quality disturbance, and the Godin Leisure-Time Exercise questionnaire will examine self-reported physical activity levels.

\section{Exercise programme}

Participants assigned to the exercise arm will be required to participate in a modular, multimodal exercise intervention with spinal isometric training (M3EP-SIT) for 12 weeks (3 months). The combined M3EP-SIT program requires participants to attend three clinic-based exercise sessions each week spanning $60 \mathrm{~min}$ in duration (including warm-up and cool-down), supervised by an accredited exercise physiologist (AEP; Exercise and Sport Science Australia) and autoregulated in collaboration with the patient (ie, adjusted based on the patient's presentation at each session). Participants will also be asked to perform the SIT program during two additional home-based exercise sessions each week spanning approximately $15 \mathrm{~min}$ in duration from week 3 onwards to allow initial familiarisation and competency assurance during the first two weeks. During the combined M3EP-SIT sessions, spinal isometric training will be provided first, followed by the modular multimodal exercise programme (table 3).

The M3EP component of the programme will be comprised of resistance, aerobic and flexibility exercises in accordance with Galvão and colleagues. ${ }^{9}$ This M3EP component is designed to minimise loads on affected

Table 3 Weekly distribution of testing, M3EP and SIT exercise sessions across the exercise intervention

\begin{tabular}{|c|c|c|c|c|c|c|c|}
\hline & Monday & Tuesday & Wednesday & Thursday & Friday & Saturday & Sunday \\
\hline Week 0 & & & & aseline testi & & & \\
\hline Weeks 1-2 & SIT M3EP & - & SIT M3EP & - & SIT M3EP & REST & REST \\
\hline
\end{tabular}

Clinic exercise sessions occur on Monday, Wednesday and Friday; home isometric exercise sessions occur on Tuesday and Thursday. Spinal isometric exercises are provided at the start of all clinic exercise sessions (following a general warm-up). Home-based SIT start from week 3 onwards to enable appropriate familiarisation and training during the first two weeks of the programme. M3EP, multimodal exercise programme (60 min); SIT, spinal isometric training (15 min). 
Table 4 Modular multimodal exercise programme for patients with bone metastases ${ }^{9} 10$

\begin{tabular}{|c|c|c|c|c|c|c|}
\hline & \multicolumn{3}{|c|}{ Resistance } & \multicolumn{2}{|c|}{ Aerobic } & \multirow{2}{*}{$\begin{array}{l}\text { Flexibility } \\
\text { Static }\end{array}$} \\
\hline Metastases site & Upper & Trunk & Lower & WB & NWB & \\
\hline Pelvis & $\sqrt{ }$ & $\sqrt{ }$ & $\sqrt{ }^{\dagger}$ & & $\sqrt{ }$ & $\sqrt{ }$ \\
\hline Lumbar spine & $\sqrt{ }$ & & $\sqrt{ }$ & & $\sqrt{ }$ & $\sqrt{ }^{\ddagger}$ \\
\hline $\begin{array}{l}\text { Thoracic spine/ } \\
\text { ribs }\end{array}$ & $\sqrt{ }^{*}$ & & $\sqrt{ }$ & $\sqrt{ }$ & $\sqrt{ }$ & $\sqrt{ }^{\ddagger}$ \\
\hline Proximal femur & $\sqrt{ }$ & $\sqrt{ }$ & $\sqrt{ }^{\dagger}$ & & $\sqrt{ }$ & $\sqrt{ }$ \\
\hline All regions & $\sqrt{ }^{*}$ & & $\sqrt{ }^{\dagger}$ & & $\sqrt{ }$ & $\sqrt{ }^{\ddagger}$ \\
\hline
\end{tabular}

*Exclusion of shoulder flexion/extension/abduction/adduction - inclusion of elbow flexion/extension.

†Exclusion of hip extension/flexion - inclusion of knee extension/flexion.

$\ddagger$ Exclusion of spine/flexion/extension/rotation.

$\checkmark$ Target exercise region.

NWB, non-weight bearing (eg, cycling); WB, weight bearing (eg, walking).

skeletal sites throughout the body. Exercise prescriptions will be modified based on the location and extent of bone metastases for all activities (table 4). Resistance exercise will be set using RM. Participants will be asked to perform six different resistance exercises using major muscle groups, subject to the location and extent of bone metastases, at 8-12 RM for three sets per exercise to achieve moderate intensity and volume. Aerobic exercise will be set using age-predicted heart rate maximum $\left(\mathrm{HR}_{\max }\right)$. Participants will undertake cardiovascular exercise using cycle ergometers, performing moderate-to-high-intensity $\left(60-85 \% \mathrm{HR}_{\max }\right)$ interval training for a total duration of $20 \mathrm{~min}$, and monitored using heart rate monitors (Polar Electro Oy, Finland). Flexibility exercise will involve static stretching of all joints considered important for function and for all muscles engaged during the session. All stretches will involve 2-4 sets per muscle group with a 30-60s hold per set. All resistance and aerobic exercise prescriptions will be progressive and periodised in accordance with each patient's individually determined physical capabilities and known contraindications.

The SIT component of the programme will comprise of exercises that isometrically load deep spinal muscles. These will be performed five times per week. Three sessions will be supervised by an AEP synonymous with the M3EP component at an exercise clinic; with an additional two sessions self-managed by the participant. This SIT component is designed to directly target and stimulate spinal lesion site(s) through muscular contraction, thus isometric exercises have been designed to activate the full spinal column due to the commonality of lesions in thoracic and lumbar regions; the feasibility of which has been demonstrated. ${ }^{60}$ The SIT program will require the participants to perform five exercises in whole and partial weight-supported prone and supine positions on the floor, while maintaining a neutral spine (isometrically) during gentle and dynamic accessory movements. If floor exercises are contraindicated for the patient due to physical restrictions, alternate seated and standing isometric exercises are also provided. All patients will be initially provided with familiarisation of breathing technique, trunk stabilisation and hip control. Basic spinal isometric exercises will first be used to ensure safe and correct technique prior to progressing to intermediate or more challenging exercises which include less stability or dynamic accessory movements. ${ }^{61}$ Isometric progression of patients from beginner to advanced exercises will be individually determined on the basis of their physical capabilities and known contraindications. An assortment of spinal isometric exercises canvassing the beginner to advanced, floor to standing spectrum is described in online supplementary table 1 , table 2 , with an assortment of sample photographs provided in the online supplementary figure for prone and supine floorbased exercises.

\section{Statistical analysis}

Data will be analysed using SPSS (IBM, Chicago, IL, USA). Normality of distribution for continuous variables will be determined using the Kolmogorov-Smirnov test and visual inspection of the data. Analyses will include standard descriptive characteristics, t-tests and two-way (group $\times$ time) repeated-measures analysis of variance (or analysis of covariance as appropriate) to examine differences between groups over time. Any data that are not normally distributed will be log-transformed or non-parametric tests will be used. For categorical variables, Pearson's $\chi^{2}$ test will be used. An alpha level of $p \leq 0.05$ will be applied to establish statistical significance. Effect sizes will also be calculated in accordance with Hopkins ${ }^{62}$ : $d \geq 0.2$ is small; $d \geq 0.6$ is moderate; $d \geq 1.2$ is large; $d \geq 2.0$ is very large. Incomplete data and missing values will be primarily managed using an intention-to-treat approach ${ }^{63}$ with multiple imputation, specifically using maximum likelihood imputation of missing values. To ensure the robustness of our findings, a secondary sensitivity analysis ${ }^{64}$ will be conducted using a complete cases approach.

\section{Dissemination}

Outcomes of this trial will be broadly disseminated through various communication channels to maximise the potential 
for further research and development. If proven to be safe, feasible and efficacious, the outcomes will form the basis of future phase II and III clinical trials. To ensure a high level of delivery to clinicians, practitioners, patients and scientists, outcomes will be disseminated through national and international clinical, patient and conference presentations, as well as publication in high-impact, peer-reviewed journals. On completion of the trial and following publication of the primary manuscripts, data requests can be submitted to the study investigators at the Exercise Medicine Research Institute (Edith Cowan University, Perth, WA, Australia).

\section{DISCUSSION}

Metastatic prostate carcinoma spreads to bone in $>80 \%$ of advanced prostate cancer cases and is presently an incurable, yet treatable stage of the disease during palliation. ${ }^{1-8}$ Consequently, these patients are provided with a suite of treatments that serve to manage pain and slow tumour progression, including ADT, radiotherapy and chemotherapy. While beneficial, these treatments produce an array of burdensome side effects leading to considerable morbidity. The effectiveness of exercise as an adjuvant therapy to minimise, manage and, in some cases, reverse the adversities of primary therapies has been demonstrated. ${ }^{14-25}$ Exercise has also shown synergistic potential with primary treatments, subsequently improving their potency; for example, enhancing chemotherapy through increased tumour vascularity to enable greater cytotoxic delivery at tumour sites. ${ }^{26-31}$ However, the direct influence of exercise on tumour biology remains largely unknown, despite many hypothesised mechanical and non-mechanical mechanisms of action. ${ }^{26}{ }^{65-70}$ To date, orthotopic animal models have provided compelling new insight into the ability of mechanical stimulation to interfere with tumour-driven remodelling in skeletal tissue containing human breast cancer cells. ${ }^{48-51}$ Given that exercise is a dose-dependent mechanical stimulant which can be safely prescribed to ]advanced prostate cancer patients with bone metastases, ${ }^{9}{ }^{10}$ it is of interest to examine whether similar modulatory interference of tumour formation and growth is achievable in humans.

This study will evaluate the feasibility, safety and preliminary efficacy of combining spinal isometric training with a modular, multimodal exercise programme to provide a non-invasive, low-cost, innovative and scalable therapy in the management of advanced prostate cancer. Specifically, this study will examine the modulatory potential of direct and targeted mechanical loading of sclerotic bone lesions through isometric exercise to deliver muscle contractile forces that may suppress localised tumour formation in spinal bone metastases, mediate systemic activity of metastatic biomarkers HIF-1 $\alpha$ (local hypoxia) and TGF- $\beta$ (tumour activity), reduce bone pain and preserve localised and surrounding musculoskeletal mass and structure. In addition, this study will also examine the multidimensional effects of exercise in advanced prostate cancer patients with bone metastases on muscle bone health, adiposity, physical fitness, functional capacity and psychosocial health. The outcomes of this study will provide innovative, new evidence that may be used to pursue larger phase II and III clinical trials to determine the efficacy of exercise on tumour suppression or regression in patients with sclerotic bone metastases secondary to prostate cancer. The outcomes of this study may also stimulate research into osteolytic or mixed bone metastases models with other primary cancer diagnoses and may also inform the development of effective pharmaceuticals for treatment. Lastly, this study presents an exercise intervention that, if effective following larger randomised controlled trials, can inevitably be delivered in clinical and community settings by exercise physiologists.

\section{Author affiliations}

${ }^{1}$ Exercise Medicine Research Institute, Edith Cowan University, Perth, Australia 2Department of Radiation Oncology, Sir Charles Gairdner Hospital, Perth, Australia ${ }^{3}$ Department of Radiation Oncology, Genesis CancerCare, Perth, Australia ${ }^{4}$ Menzies Health Institute Queensland, Griffith University, Gold Coast, Australia ${ }^{5}$ Department of Medical Oncology, St John of God Hospital, Perth, Australia ${ }^{6}$ School of Medicine, University of Notre Dame Australia, Perth, Australia ${ }^{7}$ Department of Medical Oncology, Fiona Stanley Hospital, Perth, Australia ${ }^{8}$ School of Medicine and Pharmacology, University of Western Australia, Perth, Australia

Acknowledgements The authors thank Ms Thea Richardson (Australian Prostate Cancer Research), Dr Kirk Feindel (Centre for Microscopy, Characterisation and Analysis; University of Western Australia), Mr Joey Chau (InSight Clinical Imaging), Mr Kyle Smith (Vario Health Clinic; Edith Cowan University), Mrs Claire Mason (Exercise Medicine Research Institute; Edith Cowan University) and Mr Derek Atkinson (Consumer Representative) for their contributions.

Contributors NHH, DAG, RUN and NAS developed the study concept and protocols and initiated the project. DRT, SKC, DJJ, KTF, ADR and TF assisted in further development of the protocol. NHH, DAG, RUN, DRT and SKC drafted the manuscript. NAS, DJJ, KTF, ADR and TF will provide access to patients. NHH, DAG and RUN will implement the protocol and oversee collection of data. All authors contributed and approved the final manuscript.

Funding This project is funded by Cancer Council Western Australia (Early Career Investigator Award), and supported by Exercise Sport Science Australia (Clinical Exercise Physiology Research Grant). DAG is funded by Prostate Cancer Foundation of Australia (Movember New Directions Development Award) and Cancer Council Western Australia (Research Fellowship). SKC is supported by Australian Research Council (Professorial Future Fellowship). ADR is funded by Cancer Council Western Australia (Clinical Research Fellowship).

Competing interests None declared.

Ethics approval Edith Cowan University, Human Research Ethics Committee.

Provenance and peer review Not commissioned; externally peer reviewed.

Open Access This is an Open Access article distributed in accordance with the Creative Commons Attribution Non Commercial (CC BY-NC 4.0) license, which permits others to distribute, remix, adapt, build upon this work non-commercially, and license their derivative works on different terms, provided the original work is properly cited and the use is non-commercial. See: http://creativecommons.org/ licenses/by-nc/4.0/

(c) Article author(s) (or their employer(s) unless otherwise stated in the text of the article) 2017. All rights reserved. No commercial use is permitted unless otherwise expressly granted.

\section{REFERENCES}

1. Hall CL, Bafico A, Dai J, et al. Prostate cancer cells promote osteoblastic bone metastases through wnts. Cancer Res 2005;65:7554-60.

2. Keller ET, Brown J. Prostate cancer bone metastases promote both osteolytic and osteoblastic activity. J Cell Biochem 2004;91:718-29.

3. Mundy GR. Metastasis to bone: causes, consequences and therapeutic opportunities. Nat Rev Cancer 2002;2:584-93. 
4. Keller ET, Zhang J, Cooper CR, et al. Prostate carcinoma skeletal metastases: cross-talk between tumor and bone. Cancer Metastasis Rev 2001;20:333-49.

5. Suva LJ, Washam C, Nicholas RW, et al. Bone metastasis: mechanisms and therapeutic opportunities. Nat Rev Endocrinol 2011;7:208-18.

6. Guise TA, Mohammad KS, Clines G, et al. Basic mechanisms responsible for osteolytic and osteoblastic bone metastases. Clin Cancer Res 2006;12:6213s-6.

7. Lee RJ, Saylor PJ, Smith MR. Treatment and prevention of bone complications from prostate cancer. Bone 2011;48:88-95.

8. Logothetis CJ, Lin SH. Osteoblasts in prostate cancer metastasis to bone. Nat Rev Cancer 2005;5:21-8.

9. Galvão DA, Taaffe DR, Cormie P, et al. Efficacy and safety of a modular multi-modal exercise program in prostate cancer patients with bone metastases: a randomized controlled trial. BMC Cancer 2011;11:517-23.

10. Cormie P, Newton RU, Spry N, et al. Safety and efficacy of resistance exercise in prostate cancer patients with bone metastases. Prostate Cancer Prostatic Dis 2013;16:328-35.

11. Coleman RE. Clinical features of metastatic bone disease and risk of skeletal morbidity. Clin Cancer Res 2006;12:6243s-9.

12. Carlin $\mathrm{BI}$, Andriole GL. The natural history, skeletal complications, and management of bone metastases in patients with prostate carcinoma. Cancer 2000;88:2989-94.

13. Saad F, Olsson C, Schulman CC. Skeletal morbidity in men with prostate cancer: quality-of-life considerations throughout the continuum of care. Eur Urol 2004;46:731-40.

14. Kenfield SA, Stampfer MJ, Giovannucci E, et al. Physical activity and survival after prostate cancer diagnosis in the health professionals follow-up study. J Clin Oncol 2011;29:726-32.

15. Galvão DA, Newton RU. Review of exercise intervention studies in cancer patients. J Clin Oncol 2005;23:899-909.

16. Newton RU, Galvão DA. Exercise in prevention and management of cancer. Curr Treat Options Oncol 2008;9:135-46.

17. Singh F, Newton RU, Galvão DA, et al. A systematic review of presurgical exercise intervention studies with cancer patients. Surg Oncol 2013;22:92-104.

18. Rief $\mathrm{H}$, Welzel T, Omlor $\mathrm{G}$, et al. Pain response of resistance training of the paravertebral musculature under radiotherapy in patients with spinal bone metastases-a randomized trial. BMC Cancer 2014;14:485-93.

19. Buffart LM, Galvão DA, Chinapaw MJ, et al. Mediators of the resistance and aerobic exercise intervention effect on physical and general health in men undergoing androgen deprivation therapy for prostate cancer. Cancer 2014;120:294-301.

20. Newton RU, Galvão DA. Exercise medicine for prostate cancer. European Review of Aging and Physical Activity 2013;10:41-5.

21. Cormie P, Galvão DA, Spry N, et al. Functional benefits are sustained after a program of supervised resistance exercise in cancer patients with bone metastases: longitudinal results of a pilot study. Support Care Cancer 2014;22:1537-48.

22. Hayes SC, Spence RR, Galvão DA, et al. Australian association for exercise and sport science position stand: optimising cancer outcomes through exercise. J Sci Med Sport 2009;12:428-34.

23. Buffart LM, Galvão DA, Brug J, et al. Evidence-based physical activity guidelines for cancer survivors: current guidelines, knowledge gaps and future research directions. Cancer Treat Rev 2014;40:327-40.

24. Galvão DA, Taaffe DR, Spry N, et al. Combined resistance and aerobic exercise program reverses muscle loss in men undergoing androgen suppression therapy for prostate cancer without bone metastases: a randomized controlled trial. J Clin Oncol 2010;28:340-7.

25. Galvão DA, Taaffe DR, Spry N, et al. Exercise can prevent and even reverse adverse effects of androgen suppression treatment in men with prostate cancer. Prostate Cancer Prostatic Dis 2007;10:340-6.

26. Betof AS, Lascola CD, Weitzel D, et al. Modulation of murine breast tumor vascularity, hypoxia and chemotherapeutic response by exercise. J Natl Cancer Inst 2015;107:djv040-6.

27. Hoffman P. Exercise training, tumour metabolism, tumour-host interaction and lactate shuttle theory. Front Pharmacol 2014.

28. Betof AS, Dewhirst MW, Jones LW. Effects and potential mechanisms of exercise training on cancer progression: a translational perspective. Brain Behav Immun 2013;30:S75-87.

29. Jones LW, Dewhirst MW. Therapeutic properties of aerobic training after a cancer diagnosis: more than a one-trick pony? J Natl Cancer Inst 2014;106:1-3.

30. Jones LW, Antonelli J, Masko EM, et al. Exercise modulation of the host-tumor interaction in an orthotopic model of murine prostate cancer. J Appl Physiol 2012;113:263-72.
31. Jones LW, Fels DR, West M, et al. Modulation of circulating angiogenic factors and tumor biology by aerobic training in breast cancer patients receiving neoadjuvant chemotherapy. Cancer Prev Res 2013;6:925-37.

32. Wolff G, Balke JE, Andras IE, et al. Exercise modulates redoxsensitive small GTPase activity in the brain microvasculature in a model of brain metastasis formation. PLoS One 2014:9:1-8.

33. Glass OK, Inman BA, Broadwater G, et al. Effect of aerobic training on the host systemic milieu in patients with solid tumours: an exploratory correlative study. Br J Cancer 2015;112:825-31.

34. Galvão DA, Nosaka K, Taaffe DR, et al. Endocrine and immune responses to resistance training in prostate cancer patients. Prostate Cancer Prostatic Dis 2008;11:160-5.

35. Galvão DA, Spry N, Denham J, et al. A multicentre year-long randomised controlled trial of exercise training targeting physical functioning in men with prostate cancer previously treated with androgen suppression and radiation from TROG 03.04 RADAR. Eur Urol 2014;65:856-64.

36. Galvão DA, Spry N, Taaffe DR, et al. A randomized controlled trial of an exercise intervention targeting cardiovascular and metabolic risk factors for prostate cancer patients from the RADAR trial. BMC Cancer 2009;9:419-26.

37. Galvão DA, Newton RU, Taaffe DR, et al. Can exercise ameliorate the increased risk of cardiovascular disease and diabetes associated with ADT? Nat Clin Pract Urol 2008;5:306-7.

38. Beydoun N, Bucci JA, Chin YS, et al. Prospective study of exercise intervention in prostate cancer patients on androgen deprivation therapy. J Med Imaging Radiat Oncol 2014;58:369-76.

39. Newton RU, Taaffe DR, Spry N, et al. Can exercise ameliorate treatment toxicity during the initial phase of testosterone deprivation in prostate cancer patients? is this more effective than delayed rehabilitation? BMC Cancer 2012;12:432-40.

40. Galvão DA, Taaffe DR, Spry N, et al. Acute versus chronic exposure to androgen suppression for prostate cancer: impact on the exercise response. J Urol 2011;186:1291-7.

41. Cormie P, Galvão DA, Spry N, et al. Can supervised exercise prevent treatment toxicity in patients with prostate cancer initiating androgen-deprivation therapy: a randomised controlled trial. BJU Int 2015;115:256-66.

42. Garland SN, Johnson B, Palmer C, et al. Physical activity and telomere length in early stage breast cancer survivors. Breast Cancer Res 2014;16:413-22.

43. Shammas MA. Telomeres, lifestyle, cancer, and aging. Curr Opin Clin Nutr Metab Care 2011;14:28-34.

44. Ludlow AT, Zimmerman JB, Witkowski S, et al. Relationship between physical activity level, telomere length, and telomerase activity. Med Sci Sports Exerc 2008;40:1764-71.

45. Heber S, Volf I. Effects of physical (In)activity on platelet function. Biomed Res Int 2015;2015:1-11.

46. Sharma D, Brummel-Ziedins KE, Bouchard BA, et al. Platelets in tumor progression: a host factor that offers multiple potential targets in the treatment of cancer. J Cell Physiol 2014;229:1005-15.

47. Egan K, Cooke N, Kenny D. Living in shear: platelets protect cancer cells from shear induced damage. Clin Exp Metastasis 2014;31:697-704.

48. Wang J, Lu D, Mao D, et al. Mechanomics: an emerging field between biology and biomechanics. Protein Cell 2014;5:518-31.

49. Lynch ME, Fischbach C. Biomechanical forces in the skeleton and their relevance to bone metastasis: biology and engineering considerations. Adv Drug Deliv Rev 2014;79-80:119-34.

50. Lynch ME, Brooks D, Mohanan S, et al. In vivo tibial compression decreases osteolysis and tumor formation in a human metastatic breast cancer model. J Bone Miner Res 2013;28:2357-67.

51. Chen X, Goh JC, Teoh SH, et al. Localized sclerotic bone response demonstrated reduced nanomechanical creep properties. J Mech Behav Biomed Mater 2013;17:198-208.

52. Lecouvet FE, Talbot JN, Messiou C, et al; EORTC Imaging Group. Monitoring the response of bone metastases to treatment with magnetic resonance imaging and nuclear medicine techniques: a review and position statement by the european organisation for research and treatment of cancer imaging group. Eur $\mathrm{J}$ Cancer 2014;50:2519-31.

53. Shah LM, Salzman KL. Imaging of spinal metastatic disease. Int $J$ Surg Oncol 2011;2011:1-12.

54. Tombal B, Lecouvet F. Modern detection of prostate cancer's Bone Metastasis: Is the Bone Scan Era Over? Adv Urol 2012;2012:1-8.

55. Yushkevich PA, Piven J, Hazlett HC, et al. User-guided 3D active contour segmentation of anatomical structures: significantly improved efficiency and reliability. Neuroimage 2006;31:1116-28.

56. Dunn LK, Mohammad KS, Fournier PG, et al. Hypoxia and TGFbeta drive breast cancer bone metastases through parallel signaling 
pathways in tumor cells and the bone microenvironment. PLoS One 2009;4:e6896.

57. Hart NH, Nimphius S, Spiteri T, et al. Segmental musculoskeletal examinations using Dual-Energy X-Ray absorptiometry (DXA): Positioning and analysis considerations. J Sports Sci Med 2015;14:620-6.

58. Anon. QDR for windows XP reference manual. Bedford, M.A: Hologic Inc, 2014

59. Hart NH, Nimphius S, Weber J, et al. Musculoskeletal asymmetry in football athletes: a product of limb function over time. Med Sci Sports Exerc 2016;48:1379-87.

60. Rief $\mathrm{H}$, Omlor G, Akbar M, et al. Feasibility of isometric spinal muscle training in patients with bone metastases under radiation therapy first results of a randomized pilot trial. BMC Cancer 2014;14:67-77.

61. McGill SM. Low back stability: from formal description to issues for performance and rehabilitation. Exerc Sport Sci Rev 2001;29:26-31.

62. Hopkins WG. A scale of magnitudes for effect statistics. SportScience 2002 http://www.sportsci.org/resource/stats/ effectmag.html (accessed 15 Jan 2016).

63. Gupta SK. Intention-to-treat concept: a review. Perspect Clin Res 2011;2:109-12.
64. Thabane L, Mbuagbaw L, Zhang S, et al. A tutorial on sensitivity analyses in clinical trials: the what, why, when and how. BMC Med Res Methodol 2013;13:92.

65. Jones LW, Alfano CM. Exercise-oncology research: past, present, and future. Acta Oncol 2013;52:195-215.

66. Pedersen L, Christensen JF, Hojman P. Effects of exercise on tumor physiology and metabolism. Cancer J 2015;21:111-6.

67. Secomb TW, Alberding JP, Hsu R, et al. Angiogenesis: an adaptive dynamic biological patterning problem. PLoS Comput Biol 2013;9:e1002983.

68. Siemann DW, Horsman MR. Modulation of the tumor vasculature and oxygenation to improve therapy. Pharmacol Ther 2015;153:107-24

69. Ergun M, Eyigor S, Karaca B, et al. Effects of exercise on angiogenesis and apoptosis-related molecules, quality of life, fatigue and depression in breast cancer patients. Eur J Cancer Care 2013;22:626-37.

70. Galvão DA, Taaffe DR, Spry N, et al. Enhancing active surveillance of prostate cancer: the potential of exercise medicine. Nat Rev Urol 2016;13:258-65. 